\title{
Antitumour and biological effects of letrozole and GnRH analogue as primary therapy in premenopausal women with ER and PgR positive locally advanced operable breast cancer
}

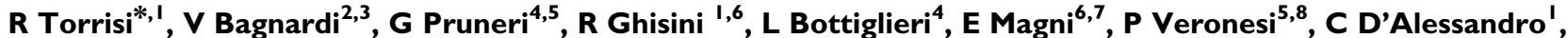
A Luini ${ }^{8}, S_{\text {Dellapasqua' }}$, G Viale ${ }^{4,5}$, A Goldhirsch $^{6}$ and M Colleoni'

'Research Unit of Medical Senology, European Institute of Oncology Milan, via Ripamonti 435, Milan 2014I, Italy; ${ }^{2}$ Division of Epidemiology and Biostatistics, European Institute of Oncology Milan, via Ripamonti 435, Milan 20 I II, Italy; ${ }^{3}$ Department of Statistics, University of Milan Bicocca, Piazza dell'Ateneo Nuovo I, Milan 20126, Italy; ${ }^{4}$ Division of Pathology, European Institute of Oncology Milan, via Ripamonti 435, Milan 20I4I, Italy; ${ }^{5}$ School of Medicine, University of Milan, via Festa del Perdono 7, Milan 20135, Italy; ${ }^{6}$ Department of Medicine, European Institute of Oncology Milan, via Ripamonti 435, Milan 2014I, Italy; ${ }^{7}$ Medical Care Unit, European Institute of Oncology Milan, via Ripamonti 435, Milan 20I 4 I, Italy; ${ }^{8}$ Division of Senology, European Institute of Oncology Milan, via Ripamonti 435, Milan 2014I, Italy
\end{abstract}

Preoperative endocrine therapy is effective in postmenopausal patients with breast cancers expressing oestrogen receptor. We investigated the activity of primary therapy with letrozole in combination with $\mathrm{GnRH}$ analogue in premenopausal women with T2-T4 N0-N2 breast cancer, whose tumours expressed oestrogen and progesterone receptors. We measured the expression of molecular factors involved in responsiveness to endocrine agents including ER $\alpha$, EGFR, HER2, MAP kinases (and phosphorylated forms) ER- $\beta$ l, both at initial biopsy and at the time of surgery. Thirty-five patients were included and 32 patients were evaluable for response. Sixteen patients $(50 \%, 95 \% \mathrm{Cl} 32-68 \%)$ obtained a partial response, 16 patients were stable. One patient showed pathological complete response (3\%, 95\% Cl 0-16\%). Response was significantly associated with younger age $(P<0.05)$ and a longer duration of treatment $(P<0.05)$. Treatment significantly decreased $E R \alpha-p-S e r{ }^{1 / 8}$ and upregulated ER- $\beta 1$, independently of response. No or negligible overexpression of EGFR was observed at baseline or after treatment in this population. Preoperative letrozole and $\mathrm{GnRH}$ analogue are effective in premenopausal women. A biological response in terms of downregulation of phosphorylated ER $\alpha$ was observed in all patients. Future investigations might focus on treatments of longer duration.

British Journal of Cancer (2007) 97, 802-808. doi:I0.I038/sj.bjc.6603947 www.bjcancer.com

Published online 21 August 2007

(C) 2007 Cancer Research UK

Keywords: preoperative; primary therapy; premenopausal; letrozole

Preoperative cytotoxic therapy has proved less effective in women with breast cancers expressing oestrogen receptor (ER) (Colleoni et al, 2004; Kaufmann et al, 2006). Endocrine therapy is a logical alternative. In postmenopausal women, neoadjuvant treatment with aromatase inhibitors showed promising results, better than those observed with tamoxifen (Eiermann et al, 2001; Miller et al, 2001; Smith et al, 2005; Abrial et al, 2006).

In premenopausal women, the use of aromatase inhibitors leads to an increase in gonadotropin secretion because of the reduced feedback of oestrogens on hypothalamus and pituitary and a subsequent stimulation of ovarian activity (Smith and Dowsett, 2003). The combination of aromatase inhibitors with GnRH analogue can obtain a complete oestrogen blockade by suppression of ovarian function and of peripheral oestrogen synthesis (Smith and Dowsett, 2003). However, limited experience has been described with aromatase inhibitors in combination with ovarian

*Correspondence: Dr R Torrisi; E-mail: rosalba.torrisi@ieo.it Received 18 April 2007; revised 30 July 2007; accepted 3I July 2007; published online 21 August 2007 function suppression in premenopausal women with advanced breast cancer (Forward et al, 2004). Aromatase inhibitors in combination with GnRH analogues have not been systematically studied as preoperative therapy in premenopausal women with locally advanced operable breast cancer.

Mechanisms underlying responsiveness and/or resistance to aromatase inhibitors are poorly understood. It has been proposed that tumour cells may escape the inhibitory effects of antioestrogens by increasing ligand-independent phosphorylation of ER, possibly as a result of an overexpression of growth factors, namely the EGFR, Her-2/neu (HER2) and the IGF-IR pathways through increased levels of mitogen-activated protein kinases (MAPKs) (Chen et al, 2002; Lannigan, 2003). The low levels of the isoform $\beta$ of ER have been associated with resistance to tamoxifen (Esslimani-Sahla et al, 2004; Hopp et al, 2004), but further data failed to show any association with response to preoperative toremifene (Cappelletti et al, 2004).

In the present study, we investigated the antitumour activity and expression of biological factors in a selected population of premenopausal patients with locally advanced breast cancer whose tumours expressed both ER and progesterone receptor $(\mathrm{PgR})$ in at least $10 \%$ of tumour cells. Given the limited knowledge on the 
endocrine effects of aromatase inhibitors in premenopausal women, we also measured sequential $17-\beta$-oestradiol levels in a subgroup of patients for whom repeated measurements were available.

\section{PATIENTS AND METHODS}

Premenopausal patients with histologically proven $\mathrm{T} 2-\mathrm{T} 4 \mathrm{~b}$ breast cancer whose tumours showed ER and PgR immunoreactivity in $\geqslant 10 \%$ of cells, consecutively admitted at the Department of Medicine of the European Institute of Oncology from January 2002 to April 2004 were enrolled in the study. All patients were submitted to a core biopsy using an 18 gauge needle of the primary tumour for histological diagnosis and assessment of biological characteristics (hormone receptor status, Ki-67 labelling index and immunoreactivity for HER2).

All patients were premenopausal as assessed by measurement of circulating oestradiol and gonadotrophins within the premenopausal range according to the reference value for each laboratory, and/or regular menses in the past 6 months. Investigations (chest $\mathrm{X}$-ray, abdomen ultrasound and bone scan) were performed to exclude distant metastasis and blood tests were performed to assess bone marrow, renal and hepatic function within 2 weeks of inclusion in the study.

The primary tumour was measured clinically by calliper in two principal diameters and by breast ultrasound and mammography. Tumour response was assessed clinically every month and by breast ultrasound and mammography after 2 and 4 months of treatment. Clinical response was defined according to WHO criteria, as $\geqslant 50 \%$ reduction of the product of two diameters of the tumour measured by calliper by the same examiner and by at least one medical imaging examination. Patients were submitted routinely to surgery after the second evaluation. A pathological complete remission (pCR) was defined as a total disappearance of the invasive tumour in both breast and axillary lymph nodes (Kuerer et al, 1999).

Endocrine therapy commenced with ovarian suppression obtained by means of a GnRH analogue, which was administered as intramuscular triptorelin $11.25 \mathrm{mg}$ every 3 months. The aromatase inhibitor letrozole $2.5 \mathrm{mg}$ per day was added when circulating oestradiol was in the postmenopausal range according to each laboratory reference value and was administered for at least 3 months. Oestradiol and gonadotrophin levels were repeated monthly during treatment with letrozole in order to verify the maintenance of ovarian function suppression.

Written informed consent was obtained from all patients. The Protocol was notified to the Ethical Committee.

This is a single institution study. All included patients had pathological evaluation performed at the EIO. Surgical specimens were extensively sampled for the evaluation of residual tumour as previously described (Colleoni et al, 2004). Immunostaining experiments for the localisation of ER (clone ID5, DakoCytomation, Glostrup, Denmark, pretreatment with EDTA, at $1: 100$ dilution) and PgR (636, DakoCytomation, EDTA, $1: 400)$, Her2/neu protein (polyclonal antibody, DakoCytomation, EDTA, $1: 800$ ) and Ki-67 antigen (MIB-1, DakoCytomation, EDTA, 1:200) were performed on consecutive tissue sections of the diagnostic biopsies and residual tumour after surgery, if any, as previously reported (Colleoni et al, 2004).

Furthermore, we also investigated the prevalence and the predictive role of the immunoreactivity for phospho-ER $\alpha\left(\mathrm{Ser}^{118}\right)$ $(\mathrm{pER} \alpha$, polyclonal antibody, Cell Signaling Technology Inc. (Danvers, MA, USA) EDTA, $1: 100$ ), ER- $\beta 1$ (PP-G5/10, DakoCytomation, citrate, $1: 20$ ), epidermal growth factor receptor (EGFR, 31G7, Zymed Laboratories (San Francisco, CA, USA) pronase, $1: 20$ ), phospho-EGFR (p-EGFR, polyclonal antibody, Cell Signaling, EDTA, 1:400), phospho-HER2 (p-HER2, polyclonal antibody,
DakoCytomation, EDTA, 1:200), phospho-p38 (p-p38MAPK, 12F8, Cell Signaling, citrate, $1: 100$ ) and phospho-p44/42 (p-p44/ 42MAPK, 20G11, Cell Signaling, citrate, 1:100) MAP-kinases in a subgroup of 27 patients for whom tumour tissue from both diagnostic biopsies and post-treatment surgical specimens was available.

In each case at least 200 neoplastic cells were evaluated at $\times 400$ magnification and the percentage of cells showing any definite nuclear (for ER, PgR and Ki-67) and membranous (for EGFR, HER2 and their phosphorylated forms) immunoreactivity was recorded.

The immunostained slides were evaluated independently by two of the authors. The thresholds for hormone receptors (ER and PgR) and Ki-67 labelling index were 10 and 20\%, respectively, according to published studies and the routine practice. The value of $20 \%$ for proliferative activity was selected based on previous data from our group indicating that this threshold significantly correlated with higher response rate to preoperative chemotherapy (Colleoni et al, 2004). For HER2, we used the FDA approved score system.

Finally, cutoffs for $\mathrm{p}-\mathrm{ER} \alpha-\mathrm{Ser}^{118}$, ER- $\beta$, EGFR, pEGFR, pHER2, p-p-38 (MAPK) and p-p44/42 (MAPK) have not been defined yet, since they have not been extensively investigated and are not used in the routine clinical practice, therefore samples containing any percentage of stained cells were considered positive.

The main end point of this study was clinical response. According to literature (Eiermann et al, 2001; Gazet et al, 2001; Smith et al, 2005), we expected to observe at least $45 \%$ of clinical responses. We adopted an optimal two-stage design, with a target rate of clinical responses of $45 \%$ deemed acceptable, and a response rate of $22 \%$ considered unacceptable.

For a 0.1 type I error and a 0.1 type II error probabilities, 17 patients were needed in the first stage and if 4 or less achieved an objective response the study had to be closed for insufficient activity. If 5 or more objective responses were observed among these initial patients, an additional 13 assessable patients had to be entered. If 10 or more objective responses were observed among the 30 assessable patients, the treatment would be considered worthy of further consideration.

The Mann - Whitney $U$-test and Fisher's exact test were used to evaluate the differences, respectively, in continuous and categorical variables between responders and non-responders.

The one-sample Wilcoxon signed-rank test and McNemar's Test were used to examine the change of expression, respectively, in continuous and categorical molecular parameters, among all tumours, between the pretreatment biopsy and surgery.

The change of expression was also evaluated in responders and non-responders, and differences between groups were also tested using the Mann-Whitney $U$-statistics.

To assess whether oestradiol levels decreases or increases during treatment, a linear mixed models for repeated-measures data were used. In the regression analysis, oestradiol levels were logarithmically transformed to reduce skewedness.

Disease-free survival (DFS) was calculated from the date of surgery to any relapse, the appearance of a second primary cancer, or death, whichever occurred first.

Estimation of the DFS was performed using the Kaplan-Meier method, and the log-rank test was used to assess the survival difference between responders and non-responders.

All analyses were performed with the SAS software (SAS Institute, Cary, NC, USA). All tests were two sided.

\section{RESULTS}

Thirty-nine consecutive patients were considered for the study and 37 were eligible. Two patients were excluded, one because of synchronous bone metastasis and one because of multifocal tumour with different receptor status. 
Among eligible patients five patients were considered not evaluable for response (two refused further treatment, one received anastrozole instead of letrozole and two did not achieve postmenopausal status within 3 months and were submitted to surgery).

Thirty-two patients were therefore considered evaluable for response. Median age was 41.5 years (range, $33-54$ years). Twentyeight patients received 3-month triptorelin and four patients received 28-day goserelin. Median duration of total treatment from initiation of GnRH analogue was 5.2 months (IQ range, 4.6-5.6), while median duration of letrozole was 4 months (IQ range, $3.4-4.4)$.

Table I Clinical and biological characteristics of tumours at baseline and at surgery

\begin{tabular}{|c|c|c|}
\hline & Trucut biopsy & Surgery \\
\hline Evaluable patients & 32 & $32^{\mathrm{a}}$ \\
\hline \multicolumn{3}{|l|}{ Clinical stage } \\
\hline $\mathrm{T} 2 / \mathrm{T} 3$ & $24 / 5$ & - \\
\hline $\mathrm{T} 4 \mathrm{~b}$ & 3 & \\
\hline $\mathrm{NO} / \mathrm{NI}$ & $7 / 25$ & \\
\hline \multicolumn{3}{|l|}{ Pathological stage } \\
\hline TO & - & 1 \\
\hline $\mathrm{TI} / \mathrm{T} 2$ & & $10 / 16$ \\
\hline $\mathrm{T} 3 / \mathrm{T} 4$ & & $4 / 1$ \\
\hline $\mathrm{NO} / \mathrm{NI}$ & & $3 / 6$ \\
\hline N2/N3 & & $12 / 5$ \\
\hline \multicolumn{3}{|l|}{ ER status } \\
\hline Negative & 0 & 1 \\
\hline $10-49 \%$ & 3 & 4 \\
\hline$\geqslant 50 \%$ & 29 & 26 \\
\hline \multicolumn{3}{|l|}{ PgR status ${ }^{\mathrm{b}}$} \\
\hline Negative & 0 & 25 \\
\hline $10-49 \%$ & 11 & 3 \\
\hline$\geqslant 50 \%$ & 21 & 2 \\
\hline \multicolumn{3}{|l|}{$\mathrm{Ki}-67$} \\
\hline$\geqslant 20 \%$ & 17 & 9 \\
\hline$<20 \%$ & 15 & 22 \\
\hline \multicolumn{3}{|c|}{ HER2 overexpression } \\
\hline $3+$ & 5 & 5 \\
\hline $2+$ & 4 & 6 \\
\hline I+/absent & 23 & 20 \\
\hline EGFR expression ${ }^{c}$ & 0 & 5 \\
\hline
\end{tabular}

${ }^{a}$ One patient was not evaluable for biological characteristics at surgery because she achieved a $\mathrm{PCR}$. ${ }^{\mathrm{b}}$ One patient with PgR $<10 \%$ was included among PgR negative. 'Tumours expressing any percentage of stained cells were considered positive.
Table 1 reports the clinical and biological characteristics of the tumours at the pretreatment biopsy and at surgery. All but one tumour maintained ER positivity at surgery, while downregulation of PgR was observed in 26 patients. Treatment also induced downregulation of proliferative activity with 9 (28\%) patients showing a Ki-67 $>20 \%$ at surgery as compared with $17(53 \%)$ patients with high proliferative activity in initial biopsy (McNemar's test $P<0.05$ ). No change was observed in HER2 overexpression.

The changes between pretreatment and the post-treatment values for PgR and Ki-67 in each patient are reported in Figure 1.

One patient (3\%) obtained a complete clinical response, which was confirmed as a pCR at pathological examination. Fifteen patients $(47 \%)$ obtained a clinical and imaging partial response giving an overall response rate of $50 \%$ (95\% CI $32-68 \%)$. Sixteen patients were stable and no patient progressed during treatment.

Breast-conserving surgery was performed in 15 patients $(47 \%)$ while 17 patients $(53 \%)$ were submitted to mastectomy. Twentytwo patients received anthracycline-containing chemotherapy as adjuvant treatment, while 10 patients continued endocrine therapy.

Median follow-up was 36 months (range, 6-51). We observed seven recurrences (one locoregional and six distant recurrences) and two deaths. Three-year DFS was 76\% (95\% CI 59-93\%) (83\% in clinical responders, $70 \%$ in non-responders, log-rank test $P$-value 0.23.).

Univariate analysis showed that clinical response was positively associated with duration of letrozole treatment which lasted 4.2 months (IQ range, 3.7-4.7) for clinical responders $v s 3.4$ months (IQ range, 2.0-5.4) for patients obtaining stable disease (MannWhitney $U$-test $P<0.05)$. Clinical response was positively associated also with age, since median age in responding patients was 39 vs 44.5 years for non-responding patients (Mann-Whitney $U$-test $P<0.05)$. Twelve $(67 \%)$ patients with $\mathrm{PgR}$ levels $\geqslant 70 \%$, corresponding to the median value of $\mathrm{PgR}$, and four patients $(29 \%)$ with $\mathrm{PgR}<70 \%$ had a clinical response (Fisher's exact test $P=0.07)$.

Twenty-three patients had repeated measurements of oestradiol at different time points during treatment. After $\mathrm{GnRH}$ analogue median oestradiol was $16 \mathrm{pg} \mathrm{ml}^{-1}$ (IQ range, 10-23). Linear mixed regression for repeated-measures data showed that there was no significant change in oestradiol levels upon treatment with letrozole (Figure 2). The oestradiol time course was not correlated with clinical response (Figure 2). Gonadotrophins were measured at the same time points of oestradiol and confirmed the suppression of $\mathrm{LH}$ and FSH.

Treatment was well tolerated and no patient discontinued therapy because of side effects. Main toxicities are reported in Table 2.

Molecular analysis was performed in 27 patients for whom tumour tissue from either pretreatment biopsy or definitive surgery was available. High levels of $\mathrm{ER} \alpha-\mathrm{p}_{-} \operatorname{Ser}^{118}$ were detected
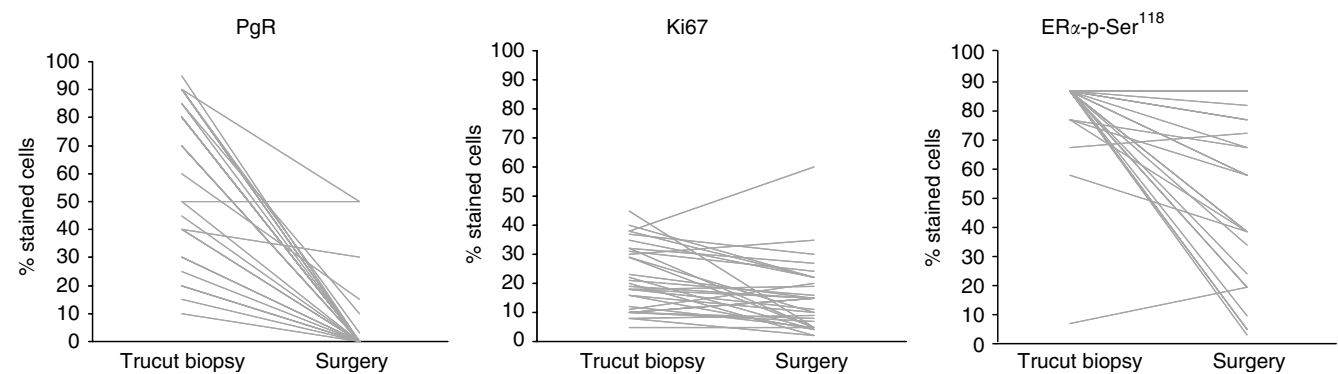

Figure I Change between pretreatment and post-treatment levels of PgR, Ki-67 and for ER $\alpha$-p-Ser ${ }^{18}$ in each patient. 


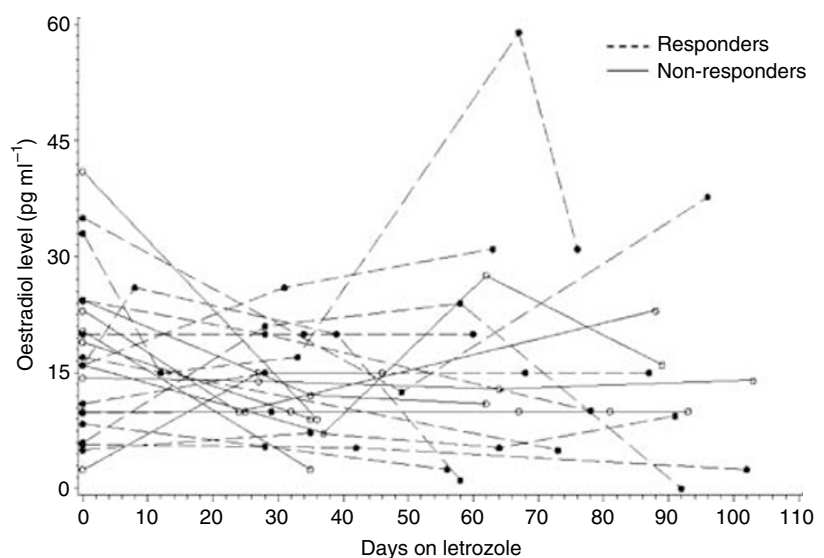

Figure 2 Time course of oestradiol levels upon treatment with letrozole according to clinical response. Time 0 is represented by the oestradiol levels obtained after $\mathrm{GnRH}$ analogue. Dashed line represents oestradiol time course in clinical responders and continuous line in non-responders.

Table 2 Main toxicities

\begin{tabular}{llc}
\hline Toxicity $(\mathbf{N}=\mathbf{3 0})$ & GI N (\%) & G2 N (\%) \\
\hline Hot flashes & $\mathbf{3}(10 \%)$ & $\mathbf{I 7}(56.7 \%)$ \\
Arthromyalgia & $\mathbf{5}(16.7 \%)$ & $\mathbf{2}(6.7 \%)$ \\
Headache & $\mathbf{4}(13 \%)$ & 0 \\
Night sweating & $\mathbf{2}(6.7 \%)$ & $\mathbf{2}(6.7 \%)$ \\
Rash & $\mathbf{I}(3.3 \%)$ & $\mathbf{I}(3.3 \%)$ \\
Nausea & $\mathbf{I}(3.3 \%)$ & $\mathbf{I}(3.3 \%)$ \\
Neurological & $\mathbf{2}(6.7 \%)$ & 0 \\
Astenia & $\mathbf{I}(3.3 \%)$ & 0 \\
Dizziness & $\mathbf{I}(3.3 \%)$ & 0 \\
\hline
\end{tabular}

in all patients and were significantly downregulated after treatment (Wilcoxon signed-rank test $P<0.0001$; Table 2). The change between pretreatment and the post-treatment values in each patient for ER $\alpha$-p-Ser ${ }^{118}$ are reported in Figure 1.

Oestrogen receptor- $\beta 1$ (ER- $\beta 1$ ) immunoreactivity was detectable in 24 patients and was significantly upregulated after treatment (Wilcoxon signed-rank test $P<0.05$; Table 3).

Neither EGFR nor its phosphorylated form (p-EGFR) was detectable in any sample in the pretreatment biopsy, while after treatment very few cells (median value 5\% range, 3-10\%) positively stained for EGFR in five surgical specimens. Phosphorylated HER2 was expressed in 10 patients (37\%) at baseline. Four out of five patients with HER2 IHC $3+$ stained positively for p-HER2 Phosphorylated-p38 (MAPK) was detected in 11 patients and p-p44/42 (MAPK) was detected in all patients except 2 at baseline and their levels remained substantially unchanged after treatment (Table 3).

Univariate analysis failed to show any association between baseline values of any molecular parameter and clinical response. No significant difference between baseline and post-treatment values were observed for any parameter among responding and not responding patients (Table 3 ).

\section{DISCUSSION}

Preoperative treatment of patients with endocrine-responsive breast cancer represents a challenge for patients with the disease, medical oncologists, surgeons and pathologists. Despite the addition of taxanes to anthracyclines, the pCR rate after preoperative chemotherapy in hormone receptor-positive tumours remains relatively low (Kaufmann et al, 2006).

Primary endocrine therapy is able to induce a high rate of objective remissions and breast-conserving surgery (Abrial et al, 2006). Preoperative endocrine therapy has been historically

Table 3 Basal levels and changes of molecular parameters after treatment according to clinical response on 27 evaluable patients

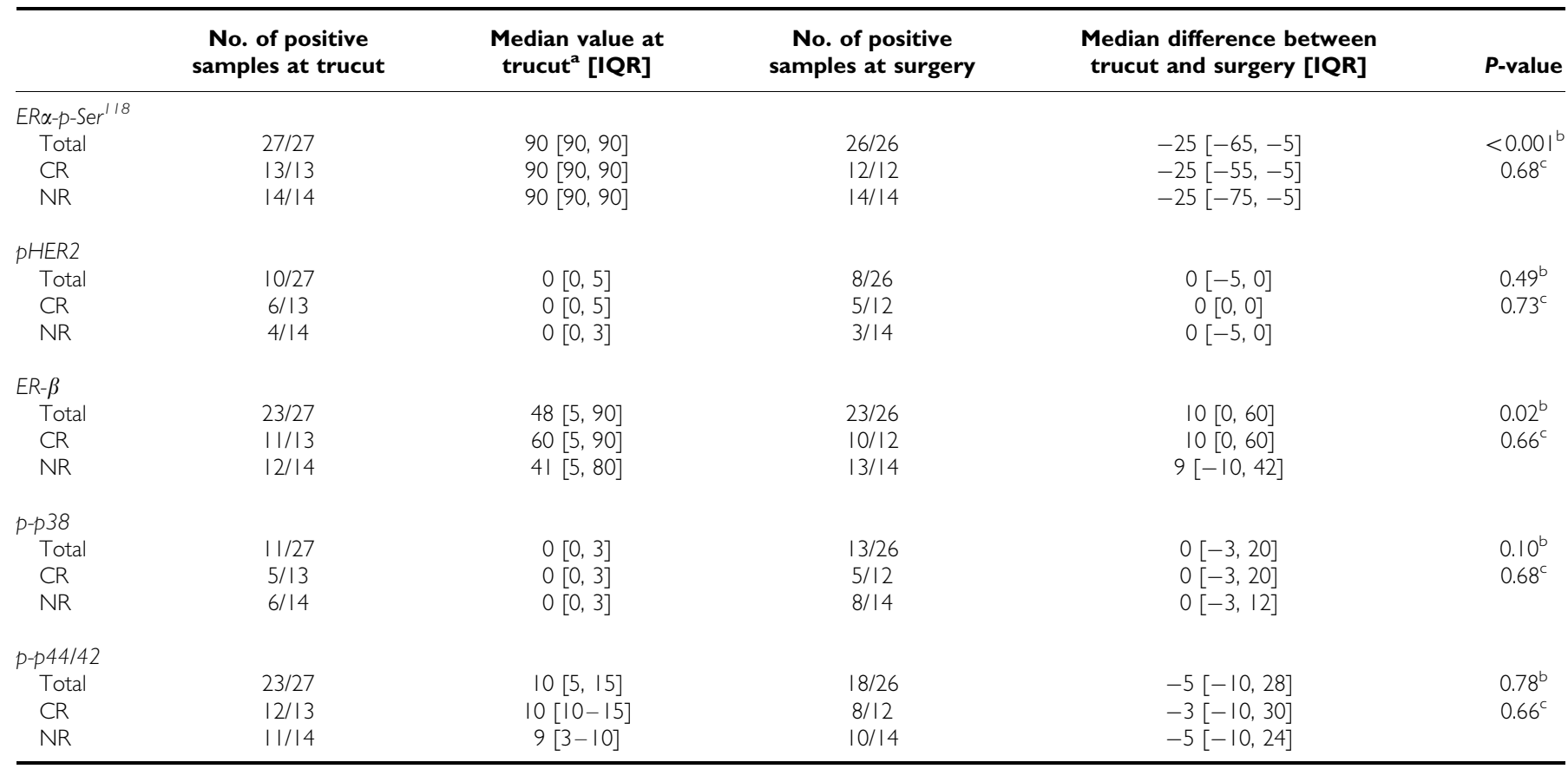

$\mathrm{CR}=$ clinical responders; $\mathrm{IQR}=$ interquartile range; $\mathrm{NR}=$ non-responders. ${ }^{\mathrm{a}}$ Values are expressed as percentage of stained cells. ${ }^{\mathrm{b}} \mathrm{Change}$ of the expression of molecular parameters among all tumours assessed by the Wilcoxon signed-rank test. ${ }^{c}$ Change of the expression of the molecular parameters between clinical responders and nonresponders assessed by the Mann-Whitney U-test. 
restricted to postmenopausal women. Data arising from very small studies have been reported in premenopausal women treated with preoperative GnRH analogue (Gazet et al, 2001; Miller et al, 2001). Aromatase inhibitors in combination with $\mathrm{GnRH}$ analogue have not been systematically studied as primary therapy in premenopausal women with hormone receptor positive locally advanced operable breast cancer. Experience in advanced disease is also limited, ranging from anecdotal reports to small phase II studies (Forward et al, 2004; Dowsett et al, 2005). The present study showed that endocrine therapy inducing a maximal oestrogen blockade is feasible in premenopausal women and effective in terms of response rate $(50 \%, 95 \%$ CI $32-68 \%)$. Results are in line with those already reported in postmenopausal women with aromatase inhibitors where the response rate ranged from 24 to $35 \%$, when considering ultrasound evaluation (Eiermann et al, 2001; Smith et al, 2005). In our study, one patient (3\%) achieved a pCR and $47 \%$ of patients were submitted to breast-conserving surgery. These figures too are comparable with those reported in randomised trials with preoperative aromatase inhibitors in postmenopausal patients. Preoperative endocrine therapy has been associated with a very low rate of PCR ranging from 1 to $8 \%$ (Kaufmann et al, 2006). The value of pCR as surrogate end point of clinical outcome in ER-positive tumours after primary chemotherapy, which was called into question in former studies, was recently confirmed by the results of a large retrospective study showing that pCR was positively associated with outcome in both ER-negative and ER-positive tumours (Ring et al, 2004; Guarneri et al, 2006), but no data after primary endocrine therapy are available. A significantly increased response rate was reported with 6 months as compared with 3 months of preoperative letrozole therapy (Paepke et al, 2006). It may therefore be that a higher pCR rate might be observed with prolonged treatment in the preoperative setting.

As expected, treatment induced a significant downregulation of both PgR expression and proliferative activity, although these were not significantly correlated with clinical response (Miller et al, 2006), although a trend for a greater reduction of Ki-67 was observed in responders.

Our results confirm a possible role for the expression of $\mathrm{PgR}$ in determining the endocrine responsiveness of the tumour. High levels of PgR were positively associated with the occurrence of a clinical response if compared with lower levels although the difference was of borderline significance.

An important issue relates to the extent of oestrogen suppression occurring upon treatment with an aromatase inhibitor in combination with a GnRH analogue in premenopausal women. Available data range from anecdotal reports to small phase II studies and include mainly the second-generation steroidal inhibitor formestane other than the non-steroidal inhibitors anastrozole and vorozole. In all reports, a further inhibition of oestradiol levels was observed after therapy with aromatase inhibitor (Dowsett et al, 1992, 1999; Celio et al, 1999; Forward et al, 2004). In our study, we evaluated the levels of oestradiol at different time points during the study to determine the hormonal status. We acknowledge that our data are limited because of the heterogeneity of time points of the determinations and the multiple laboratories employed, other than the lack of adequately sensitive test for measuring oestradiol. We found that oestradiol levels were maintained in the postmenopausal range. We observed a slight and not significant further suppression of oestradiol after letrozole administration only in 13 patients out of 23. Whether the extent of oestradiol suppression may affect the clinical response is currently unknown. Although our data in a limited number of patients failed to show any correlation between oestradiol time course and clinical response, the issue of oestrogen suppression in premenopausal women receiving aromatase inhibitors needs to be further addressed, given these drugs are currently investigated in the adjuvant setting in large phase III trials. On the other hand, two patients failed to reach a full-ovarian function suppression within 3 months after the first administration of 3-month triptorelin as documented by persisting menstrual cycle and oestradiol levels within the range of premenopause and were submitted to surgery. The failure of the 3-month GnRH analogue formulation in achieving ovarian function suppression has been previously reported either in breast and in prostate cancer patients (Yri et al, 2006; Schmid et al, 2007).

Few data are available on the molecular changes occurring in vivo after neoadjuvant therapy with aromatase inhibitors and their predictive value on clinical response. Our study population was highly selected according to the expression of both steroid hormone receptors. Molecular analysis showing high baseline levels of $\mathrm{ER} \alpha$-p-Ser ${ }^{118}$ and the absent or negligible expression of EGFR and HER2 in all patients emphasise the endocrine-sensitive nature of our population.

Almost all available data on mechanisms of resistance to aromatase inhibitors are derived from preclinical models using long-term oestrogen-deprived (LTED) cells which supposedly mimic the oestrogen depletion seen clinically with letrozole (Brodie et al, 2005; Dowsett et al, 2005). In these models, an upregulation of the ER $\alpha$-p-Ser ${ }^{118}$ following an activation of growth factor pathway signalling (EGFR, IGFR) and the activation of MAP kinase has been demonstrated when tumours started to grow despite letrozole (Chen et al, 2006).

$\mathrm{Ser}^{118}$ represents the major site of phosphorylation of ER upon binding with oestradiol (Lannigan, 2003). Baseline levels of ER $\alpha$-pSer ${ }^{118}$ have been positively associated with favourable tumour characteristics, with a better outcome after therapy with tamoxifen and with expression of PgR (Murphy et al, 2004a, b). It has been hypothesised that, as distinct from what occurs in vitro, ER $\alpha$-p$\mathrm{Ser}^{118}$ is a marker of a functional ligand-dependent pathway in primary breast tumours and is involved in the mechanisms by which the oestradiol/ER $\alpha$ complex regulates downstream signalling (Murphy et al, 2004b).

We may speculate that the downregulation of the ER $\alpha$-p-Ser ${ }^{118}$ we observed after treatment is a consequence of the suppressed levels of circulating oestrogens, suggesting the occurrence of a reduced oestrogen-induced transcription, leading finally to a clinical response. In a previous study with gefitinib plus or minus anastrozole, the decrease of $\mathrm{ER} \alpha-\mathrm{p}-\mathrm{Ser}^{118}$ was associated with clinical response (Polychronis et al, 2005). Therefore, we might have expected that non-responders should not experience a decrease of $\mathrm{ER} \alpha-\mathrm{p}-\mathrm{Ser}^{118}$, but rather an increased phosphorylation as a consequence of a ligand-independent activation which is a putative mechanism of endocrine resistance (Brodie et al, 2005; Chen et al, 2006). However, in our study no difference in the extent of the decreased phosphorylation was observed in responders or non-responders, and we saw no increase in EGFR or HER2 expression or in phosphorylated MAP kinase activity in the clinical non-responders. It is possible that our nonresponders were not inherently resistant, but merely not treated for long enough.

Our results on the correlation between biomarkers and clinical response, however, should be considered with caution given the small number of patients included in this analysis.

The significance and the role of ER- $\beta$ and the interaction with ER- $\alpha$ are unclear. The ratio ER $\alpha:$ ER- $\beta$ within tumour cells has been hypothesised to play a functional role in cell growth (Shaw et al, 2006). Conflicting data have been reported on the role of ER- $\beta$ either in vitro and in vivo, in that results from preclinical and clinical studies have associated ER- $\beta$ either positively (protein expression) or negatively (mRNA) with prognosis and response to endocrine treatment (Cappelletti et al, 2004; Saji et al, 2005). The results of our study, showing a significant upregulation of ER- $\beta$ after treatment irrespective of clinical response, do not help to get further insight into this issue. 
In conclusion, the results of the present study indicate that therapy with aromatase inhibitors, in combination with a GnRH analogue, is safe and effective in premenopausal women with locally advanced operable breast cancer. A biological response, in terms of downregulation of the oestrogenic signalling, was observed in all patients, and clinical response in $50 \%$ of the patients. The trend to improved response rate observed with longer duration in the present study and in previously published studies support the development of tailored endocrine therapy of longer duration in selected populations of premenopausal patients with endocrine-responsive tumours.

\section{ACKNOWLEDGEMENTS}

We thank the patients, nurses, data managers and physicians at the European Institute of Oncology. Special acknowledgement to Professor Alan S Coates for relevant input to the article.

\section{REFERENCES}

Abrial C, Mouret-Reynier MA, Cure H, Feillel V, Leheurteur M, Lemery S, Le BG, Durando X, Dauplat J, Chollet P (2006) Neoadjuvant endocrine therapy in breast cancer. Breast 15: $9-19$

Brodie A, Jelovac D, Sabnis G, Long B, Macedo L, Goloubeva O (2005) Model systems: mechanisms involved in the loss of sensitivity to letrozole. J Steroid Biochem Mol Biol 95: 41-48

Cappelletti V, Celio L, Bajetta E, Allevi A, Longarini R, Miodini P, Villa R, Fabbri A, Mariani L, Giovanazzi R, Galante E, Greco M, Grazia DM (2004) Prospective evaluation of estrogen receptor-beta in predicting response to neoadjuvant antiestrogen therapy in elderly breast cancer patients. Endocr Relat Cancer 11: 761-770

Celio L, Martinetti A, Ferrari L, Buzzoni R, Mariani L, Miceli R, Seregni E, Procopio G, Cassata A, Bombardieri E, Bajetta E (1999) Premenopausal breast cancer patients treated with a gonadotropin-releasing hormone analog alone or in combination with an aromatase inhibitor: a comparative endocrine study. Anticancer Res 19: 2261-2268

Chen D, Washbrook E, Sarwar N, Bates GJ, Pace PE, Thirunuvakkarasu V, Taylor J, Epstein RJ, Fuller-Pace FV, Egly JM, Coombes RC, Ali S (2002) Phosphorylation of human estrogen receptor alpha at serine 118 by two distinct signal transduction pathways revealed by phosphorylationspecific antisera. Oncogene 21: 4921-4931

Chen S, Masri S, Wang X, Phung S, Yuan YC, Wu X (2006) What do we know about the mechanisms of aromatase inhibitor resistance? J Ster Biochem Mol Biol 102: 232-240

Colleoni M, Viale G, Zahrieh D, Pruneri G, Gentilini O, Veronesi P, Gelber RD, Curigliano G, Torrisi R, Luini A, Intra M, Galimberti V, Renne G, Nole F, Peruzzotti G, Goldhirsch A (2004) Chemotherapy is more effective in patients with breast cancer not expressing steroid hormone receptors: a study of preoperative treatment. Clin Cancer Res 10: $6622-6628$

Dowsett M, Doody D, Miall S, Howes A, English J, Coombes RC (1999) Vorozole results in greater oestrogen suppression than formestane in postmenopausal women and when added to goserelin in premenopausal women with advanced breast cancer. Breast Cancer Res Treat 56: 25-34

Dowsett M, Johnston S, Martin LA, Salter J, Hills M, Detre S, Gutierrez MC, Mohsin SK, Shou J, Allred DC, Schiff R, Osborne CK, Smith I (2005) Growth factor signalling and response to endocrine therapy: the Royal Marsden Experience. Endocr Relat Cancer 12(Suppl 1): S113-S117

Dowsett M, Mehta A, King N, Smith IE, Powles TJ, Stein RC, Coombes RC (1992) An endocrine and pharmacokinetic study of four oral doses of formestane in postmenopausal breast cancer patients. Eur J Cancer 28: $415-420$

Eiermann W, Paepke S, Appfelstaedt J, Llombart-Cussac A, Eremin J, Vinholes J, Mauriac L, Ellis M, Lassus M, Chaudri-Ross HA, Dugan M, Borgs M (2001) Preoperative treatment of postmenopausal breast cancer patients with letrozole: a randomized double-blind multicenter study. Ann Oncol 12: 1527-1532

Esslimani-Sahla M, Simony-Lafontaine J, Kramar A, Lavaill R, Mollevi C, Warner M, Gustafsson JA, Rochefort H (2004) Estrogen receptor beta (ER beta) level but not its ER betacx variant helps to predict tamoxifen resistance in breast cancer. Clin Cancer Res 10: 5769-5776

Forward DP, Cheung KL, Jackson L, Robertson JF (2004) Clinical and endocrine data for goserelin plus anastrozole as second-line endocrine therapy for premenopausal advanced breast cancer. $\mathrm{Br} J$ Cancer 90: $590-594$

Gazet JC, Ford HT, Gray R, McConkey C, Sutcliffe R, Quilliam J, Makinde V, Lowndes S, Coombes RC (2001) Estrogen-receptor-directed neoadjuvant therapy for breast cancer: results of a randomised trial using formestane and methotrexate, mitozantrone and mitomycin C (MMM) chemotherapy. Ann Oncol 12: 685-691
Guarneri V, Broglio K, Kau SW, Crsitofanilli M, Buzdar AU, Valero V, Bucholz T, Meric F, Middl eton L, Hortobagyi GN, Gonzalez-Angulo AM (2006) Prognostic value of pathologic complete response after primary chemotherapy in relation to hormone receptor status and other factors. J Clin Oncol 24: 1037 - 1044

Hopp TA, Weiss HL, Parra IS, Cui Y, Osborne CK, Fuqua SA (2004) Low levels of estrogen receptor beta protein predict resistance to tamoxifen therapy in breast cancer. Clin Cancer Res 10: 7490-7499

Kaufmann M, Hortobagyi GN, Goldhirsch A, Scholl S, Makris A, Valagussa P, Blohmer JU, Eiermann W, Jackesz R, Jonat W, Lebeau A, Loibl S, Miller W, Seeber S, Semiglazov V, Smith R, Souchon R, Stearns V, Untch M, von MG (2006) Recommendations from an international expert panel on the use of neoadjuvant (primary) systemic treatment of operable breast cancer: an update. J Clin Oncol 24: $1940-1949$

Kuerer HM, Newman LA, Smith TL, Ames FC, Hunt KK, Dhingra K, Theriault RL, Singh G, Binkley SM, Sneige N, Buchholz TA, Ross MI, McNeese MD, Buzdar AU, Hortobagyi GN, Singletary SE (1999) Clinical course of breast cancer patients with complete pathologic primary tumor and axillary lymph node response to doxorubicin-based neoadjuvant chemotherapy. J Clin Oncol 17: 460-469

Lannigan DA (2003) Estrogen receptor phosphorylation. Steroids 68: $1-9$

Miller WR, Dixon JM, Cameron DA, Anderson TJ (2001) Biological and clinical effects of aromatase inhibitors in neoadjuvant therapy. J Steroid Biochem Mol Biol 79: 103-107

Miller WR, White S, Dixon JM, Murray J, Renshaw L, Anderson TJ (2006) Proliferation, steroid receptors and clinical/pathological response in breast cancer treated with letrozole. Br J Cancer 94: 1051-1056

Murphy L, Cherlet T, Adeyinka A, Niu Y, Snell L, Watson P (2004a) Phospho-serine-118 estrogen receptor-alpha detection in human breast tumors in vivo. Clin Cancer Res 10: 1354-1359

Murphy LC, Niu Y, Snell L, Watson P (2004b) Phospho-serine-118 estrogen receptor-alpha expression is associated with better disease outcome in women treated with tamoxifen. Clin Cancer Res 10: $5902-5906$

Paepke S, Jacobs VR, Paepke D, Euler U, Blohmer JU, Warm M, Ohlinger R, Fischer T, Kiechle M, Harbeck N (2006) Critical appraisal of primary systemic endocrine therapy in receptor-positive postmenopausal breast cancer: an update. Onkologie 29: 210-217

Polychronis A, Sinnett HD, Hadjiminas D, Singhal H, Mansi JL, Shivapatham D, Shousha S, Jiang J, Peston D, Barrett N, Vigushin D, Morrison K, Beresford E, Ali S, Slade MJ, Coombes RC (2005) Preoperative gefitinib $v s$ gefitinib and anastrozole in postmenopausal patients with oestrogen-receptor positive and epidermal-growth-factorreceptor-positive primary breast cancer: a double-blind placebocontrolled phase II randomised trial. Lancet Oncol 6: 383-391

Ring AE, Smith IE, Ashley S, Fulford LG, Lakhani SR (2004) Oestrogen receptor status, pathological complete response and prognosis in patients receiving neoadjuvant chemotherapy for early breast cancer. Br J Cancer 91: $2012-2017$

Saji S, Hirose M, Masakazu T (2005) Clinical significance of estrogen receptor $\beta$ in breast cancer. Cancer Chemother Pharmacol 56: s21-s26

Schmid P, Untch M, Kossé V, Bondar G, Vassilijev L, Tarutinov V, Lehmann U, Maubach L, Meurer J, Wallwiener D, Possinger K (2007) Leuprorelin acetate every 3-months Depot vs cyclophosphamide, methotrexate and fluorouracil as adjuvant treatment in premenopausal patients with node-positive breast cancer: the TABLE study. J Clin Oncol 25: $2509-2515$ 
Preoperative letrozole and $\mathrm{GnRH}$ in premenopausal breast cancer

Shaw LE, Sadler AJ, Pugazhendhi D, Darbre PD (2006) Changes in oestrogen receptor-alpha and -beta during progression to acquired resistance to tamoxifen and fulvestrant (Faslodex, ICI 182 780) in MCF7 human breast cancer cells. J Steroid Biochem Mol Biol 99: 19-32

Smith IE, Dowsett M (2003) Aromatase inhibitors in breast cancer. N Engl J Med 348: $2431-2442$

Smith IE, Dowsett M, Ebbs SR, Dixon JM, Skene A, Blohmer JU, Ashley SE, Francis S, Boeddinghaus I, Walsh G (2005) Neoadjuvant treatment of postmenopausal breast cancer with anastrozole, tamoxifen, or both in combination: the immediate preoperative anastrozole, tamoxifen, or combined with tamoxifen (IMPACT) multicenter double-blind randomized trial. $J$ Clin Oncol 23: $5108-5116$

Yri OE, Bjoro T, Fossa SD (2006) Failure to achieve castration levels in patients using leuprolide acetate in locally advanced prostate cancer. Eur Urol 49: 54-58 